\title{
Discrete Chebyshev Polynomials for Solving Fractional Variational Problems
}

\author{
Fakhrodin Mohammadi $^{1, *}$, Leila Moradi ${ }^{2}$, Dajana Conte ${ }^{2}$ \\ ${ }^{1}$ Department of Mathematics, University of Hormozgan, Iran \\ ${ }^{2}$ Department of Mathematics, University of Salerno, Italy
}

\begin{abstract}
In the current study, a general formulation of the discrete Chebyshev polynomials is given. The operational matrix of fractional integration for these discrete polynomials is also derived. Then, a numerical scheme based on the discrete Chebyshev polynomials and their operational matrix has been developed to solve fractional variational problems. In this method, the need for using Lagrange multiplier during the solution procedure is eliminated. The performance of the proposed scheme is validated through some illustrative examples. Moreover, the obtained numerical results were compared to the previously acquired results by the classical Chebyshev polynomials. Finally, a comparison for the required CPU time is presented, which indicates more efficiency and less complexity of the proposed method.
\end{abstract}

Keywords Discrete Chebyshev polynomials, Chebyshev polynomials, Operational matrix, Fractional variational problems.

AMS 2010 subject classifications 49M99, 26A33

DOI:10.19139/soic-2310-5070-991

\section{Introduction}

In the last two decades, numerical approaches based on orthogonal polynomials have been frequently used to approximate solution of fractional differential and integral equations $[1,5,4,3,2]$. The key characteristic of these approaches is that, by using the orthogonal properties of the polynomials and the typical spectral methods, they reduce such fractional equations to a system of algebraic equations which can be solved more easier. According to the defined inner product in the solution space, orthogonal polynomials are usually classified into two main classes: continuous and discrete. For continuous orthogonal polynomials such as Legendre, Chebyshev, Hermite, and Laguerre one has to evaluate an integral in the inner product, whereas discrete orthogonal polynomials come with a discrete scalar product and hence the integral becomes a sum[6, 7]. Although continuous orthogonal polynomials have been more frequently used to approximate solution of functional equations, there are some advantages of using discrete orthogonal polynomials. For example, by using discrete orthogonal polynomials, the Fourier coefficients can be calculated with the aid of a summation and the obtained coefficients are exact. Consequently, in comparison to continouse cases, implimentation of discrete orthogonal polynomials is more efficient and less complex $[8,9]$. Moreover, there is a close connection between stochastic processes and discrete orthogonal polynomials which motivated many researchers to consider them to solve stochastic differential equations [10,8].

In the field of optimization theory, calculus of variations concerns with the problem of optimizing a realvalued functional over a set of functions $[11,12,13,14]$. The functional is usually defined as definite integrals involving functions and their derivatives. The development of the calculus of variations starts with Johann Bernoulli *Correspondence to: Fakhrodin Mohammadi (Email: fmohammadi62@hotmail.com). Department of Mathematics, University of
Hormozgan, P. O. Box 3995 Bandar Abbas, Iran.

ISSN 2310-5070 (online) ISSN 2311-004X (print)

Copyright (C) 2021 International Academic Press 
and his well-known "brachistochrone" problem. Thereafter, this subject was developed by the works of Jakob Bernoulli, Newton, Leibniz, Euler, Lagrange, Legendre, Jacobi, Weierstrass and Hilbert[13]. Recently, the calculus of variations has been found many applications in areas such as quantum mechanics, medicine, economics, aerodynamics, environmental engineering and biology[17, 16, 15, 11, 18, 19, 20]. As a generalization of calculus of variations, the fractional calculus of variations (FCV) deals with variational functionals depending on fractional derivatives instead of integer ones[11]. The subject of FCV was introduced in 1996 with the work of Riewe [18]. He used fractional derivatives to formulate the problem of calculus of variations and obtained the respective Euler-Lagrange equations. Nowadays, FCV is under development due to its many applications in physics and engineering[17, 16, 15, 11, 19, 21].

The main purpose of this paper is to present a comparitive study of numerical solution of the following fractional variational problems:

$$
\operatorname{Min.} J(x)=\int_{a}^{b} \mathcal{F}\left(t, x(t),{ }_{0}^{C} D_{t}^{\nu} x(t), u(t)\right) d t,
$$

in which the variable $u(t)$ is described by

$$
u(t)=\int_{a}^{t} \mathcal{K}\left(s, x(s),{ }_{0}^{C} D_{t}^{\nu} x(s)\right) d s, \quad a \leq t \leq b,
$$

subject to the following boundary conditions

$$
x(a)=\alpha, x(b)=\beta .
$$

The functions $\mathcal{F}$ and $\mathcal{K}$ are continuously differentiable, $x$ is absolutely continuous functions, such that its fractional derivative of order $\nu$, i.e. ${ }_{0}^{C} D_{t}^{\nu} x(t)$, exists and is continuous on $[a, b]$. First, the general formulation of DCPs and their properties are investigated briefly. Then, DCPs and their operational matrix of fractional integration are used to approximate solution of FVP (1)-(3). The obtained numerical results and the required CPU time were compared to the previously acquired results by the classical Chebyshev polynomials in Ref. [16]. The comparison reveals that presented DCPs method is more efficient and less complex than the Chebyshev polynomials method.

The remainder of the paper is organized as follows: In Section 2, some basic definitions and notations of Riemann-Liouville and Caputo fractional operators are introduced. Section 3 is devoted to definition of discrete Chebyshev polynomials and their properties. Section 4 deals with the numarical solution of FVPs. In Section 5, some illustrative examples are given to verify superiority of the discrete Chebyshev polynomials. Finally, some concluding remarks are given in Section 6.

\section{Preliminaries}

The most frequently used definitions for fractional integral and derivative are the Caputo and Riemann-Liouville, which can be defined as follows [24, 23, 22].

\section{Definition 1}

The fractional integration of order $\nu \geq 0$ in the Riemann-Liouville sense is defined as

$$
\left(I^{\nu} f\right)(t)= \begin{cases}f(t), & \nu=0, \\ \frac{1}{\Gamma(\nu)} \int_{0}^{t} \frac{f(s) d s}{(t-s)^{1-\nu}}, & \nu>0 .\end{cases}
$$


Some practical properties of the fractional operator $I^{\nu}$ can be easily verified as follow:

$$
\begin{aligned}
& { }_{0} I_{t}^{\nu_{1}}\left({ }_{0} I_{t}^{\nu_{2}} f(t)\right)={ }_{0} I_{t}^{\nu_{2}}\left({ }_{0} I_{t}^{\nu_{1}} f(t)\right), \quad \nu_{1}, \nu_{2} \geq 0, \\
& { }_{0} I_{t}^{\nu_{1}}\left({ }_{0} I_{t}^{\nu_{2}} f(t)\right)={ }_{0} I_{t}^{\nu_{1}+\nu_{2}} f(t), \quad \nu_{1}, \nu_{2} \geq 0, \\
& { }_{0} I_{t}^{\nu} t^{r}=\frac{\Gamma(r+1)}{\Gamma(r+\nu+1)} t^{\nu+r}, \nu \geq 0, r>-1 .
\end{aligned}
$$

Definition 2

Let $\nu$ be a real positive number. The Caputo fractional derivative operator of order $\nu$, i.e. ${ }_{0}^{C} D_{t}^{\nu}$, could be defined as follow:

$$
{ }_{0}^{C} D_{t}^{\nu} f(t)= \begin{cases}\frac{1}{\Gamma(n-\nu)} \int_{0}^{t} \frac{f^{(n)}(s)}{(t-s)^{\nu-n+1}} d s, t>0, & 0 \leq n-1<\nu<n, \\ \frac{d^{n} f(t)}{d t^{n}}, & \nu=n \in \mathbb{N} .\end{cases}
$$

where $n$ is an integer, $t>0$.

Also, the following properties of the Caputo fractional operator ${ }_{0}^{C} D_{t}^{\nu}$ can be easily verified:

$$
\begin{gathered}
{ }_{0}^{C} D_{t}^{\nu}\left({ }_{0} I_{t}^{\nu} f(t)\right)=f(t) . \\
{ }_{0} I_{t}^{\nu}\left({ }_{0}^{C} D_{t}^{\nu} f(t)\right)=f(t)-\sum_{j=0}^{n-1} f^{(j)}\left(0^{+}\right) \frac{t^{j}}{j !}, \quad t>0 . \\
{ }_{0}^{C} D_{t}^{\nu} t^{r}= \begin{cases}0, & \text { for } r \in \mathbb{N} \cup\{0\} \text { and } r<\nu, \\
\frac{\Gamma(r+1)}{\Gamma(r-\nu+1)} t^{r-\nu}, & \text { otherwise. }\end{cases}
\end{gathered}
$$

\section{Discrete Chebyshev polynomials}

The discrete Chebyshev polynomials belong to a rich family of orthogonal polynomials which introduced by Chebyshev. In this section, we restrict our attention to the basic definition, formula and properties of these polynomials. For more details and some applications of these polynomials one can refer to Refs. [25, 26].

\section{Definition 3}

Let $N$ be a positive integer number. The discrete Chebyshev polynomials $\mathcal{T}_{n, N}(x)$ may be defined as follow:

$$
\mathcal{T}_{n, N}(x)=\sum_{k=0}^{n}(-1)^{k}\left(\begin{array}{c}
n+k \\
n
\end{array}\right)\left(\begin{array}{c}
N-k \\
n-k
\end{array}\right)\left(\begin{array}{l}
x \\
k
\end{array}\right), n=0,1, \ldots, N .
$$

\section{Orthogonality:}

The set of discrete Chebyshev polynomials $\left\{\mathcal{T}_{n, N}, n=0,1, \ldots, N\right\}$ is orthogonal on the interval $[0, N]$ with respect to the following discret norm:

$$
\langle f, g\rangle=\sum_{r=0}^{N} f(r) g(r)
$$


Moreover, the orthogonality condition for these polynomials is as follow:

$$
\left\langle\mathcal{T}_{m, N}, \mathcal{T}_{n, N}\right\rangle=\sum_{r=0}^{N} \mathcal{T}_{m, N}(r) \mathcal{T}_{n, N}(r)=\mu_{n} \delta_{m n},
$$

where $\delta_{m n}$ is the Kronecker delta and $\mu_{n}$ can be defined as:

$$
\mu_{n}=\frac{(N+n+1) !}{(2 n+1)(N-n) !(n !)^{2}}
$$

\section{Recurrence relation:}

The set of discrete Chebyshev polynomials $\left\{\mathcal{T}_{n, N}, n=0,1, \ldots, N\right\}$ can be determined with the aid of the following recurrence formulae:

$$
\alpha_{n} \mathcal{T}_{n+1, N}(x)=\frac{(N-k)\left(\alpha_{n}+\beta_{n}-x\right)}{k+1} \mathcal{T}_{n, N}(x)-\frac{(N-k+1)(N-k) \beta_{n}}{k(k+1)} \mathcal{T}_{n-1, N}(x), \quad n=0,1, \ldots, N
$$

where $\mathcal{T}_{0, N}(x)=1, \mathcal{T}_{1, N}(x)=N-2 x$ and

$$
\alpha_{n}=\frac{(N-n)(n+1)^{2}}{(2 n+1)(2 n+2)}, \quad \beta_{n}=\frac{N^{2}(N+n+1)}{2 n(2 n+1)} .
$$

\section{Explicit formula:}

By using the relation (4), the discrete Chebyshev polynomial $\mathcal{T}_{n, N}$ can be written as follow:

$$
\begin{aligned}
& \mathcal{T}_{n, N}(x)=\sum_{k=0}^{n} \frac{(-1)^{k}\left(\begin{array}{c}
n+k \\
n
\end{array}\right)\left(\begin{array}{c}
N-k \\
n-k
\end{array}\right)}{k !} x(x-1) \ldots(x-k+1) \\
& =\sum_{k=0}^{n} \frac{(-1)^{k}\left(\begin{array}{c}
n+k \\
n
\end{array}\right)\left(\begin{array}{c}
N-k \\
n-k
\end{array}\right)}{k !} \sum_{i=0}^{k} s(k, i) x^{i}, \quad n=0,1, \ldots N,
\end{aligned}
$$

in which $s(k, i)$ are the Stirling numbers of the first kind [27, 28]. Therefore, the analytical form of $\mathcal{T}_{n, N}(x)$ can be derived as follow:

$$
\mathcal{T}_{n, N}(x)=\sum_{k=0}^{n} a_{j, n} x^{k}, \quad n=0,1, \ldots N
$$

where

$$
a_{j, n}=\sum_{r=j}^{n} \frac{(-1)^{r}\left(\begin{array}{c}
n+r \\
n
\end{array}\right)\left(\begin{array}{c}
N-r \\
n-r
\end{array}\right) s(r, i)}{r !} .
$$

\section{Shifted discrete Chebyshev polynomials:}

In order to use the discrete Chebyshev polynomials on the interval $[0,1]$ we define the shifted discrete Chebyshev polynomials (SDCPs) by introducing change of variable $x=N t$. Let $n$th shifted discrete Chebyshev polynomial, i.e. $\mathcal{T}_{n, N}(N t)$, is denoted by $\mathfrak{T}_{n, N}(t)$. Then, the set of SDCPs $\left\{\mathfrak{T}_{n, N}, n=0,1, \ldots, N\right\}$ are orthogonal on the interval 
$[0,1]$ with respect to the following discrete norm:

$$
\langle f, g\rangle_{*}=\sum_{r=0}^{N} f\left(\frac{r}{N}\right) g\left(\frac{r}{N}\right) .
$$

Also, the orthogonality condition for SDCPs can be defined as follow:

$$
\left\langle\mathfrak{T}_{m, N}(t), \mathfrak{T}_{n, N}(t)\right\rangle_{*}=\sum_{r=0}^{N} \mathfrak{T}_{m, N}\left(\frac{r}{N}\right) \mathfrak{T}_{n, N}\left(\frac{r}{N}\right)=\mu_{n} \delta_{m n}
$$

where $\mu_{n}$ is defined in relation (6).

\section{Function approximation:}

Any function $f(t)$, defined over the interval $[0,1]$ may be expanded by the SDCPs as

$$
f(t) \simeq \sum_{i=0}^{N} c_{i} \mathfrak{T}_{i, N}(t)=C^{T} \Psi(t)
$$

where $C$ and $\Phi(x)$ are $(N+1)$ vectores given by

$$
\begin{gathered}
C^{T}=\left[c_{0}, c_{1}, \ldots, c_{N}\right]^{T}, \\
\Psi(t)=\left[\mathfrak{T}_{0, N}(t), \mathfrak{T}_{1, N}(t), \ldots, \mathfrak{T}_{N, N}(t)\right]^{T},
\end{gathered}
$$

and the coefficients $c_{i}$ can be derived as follow

$$
c_{i}=\frac{\left\langle f(t), \mathfrak{T}_{i, N}(t)\right\rangle_{*}}{\mu_{i}}, \quad i=0,1, \ldots N
$$

\section{Convergence analysis:}

The discrete Chebyshev polynomials $\mathcal{T}_{n, N}(x)$ are special case of the well-known Hahn polynomials $Q_{n}(x ; \alpha, \beta, N)$ with $\alpha=\beta=0$ [9]. The following Theorem provides conditions that ensure the series expansion of a function by the discrete Chebyshev polynomials converges.

\section{Theorem 1}

The series expansion $\sum_{k=0}^{n} \frac{\left\langle f, \mathcal{T}_{k, N}\right\rangle}{\left\langle\mathcal{T}_{k, N}, \mathcal{T}_{k, N}\right\rangle} \mathcal{T}_{k, N}(x)$ of a function $f$ by the discrete Chebyshev polynomials converges pointwise, if the series expansionof the function $f$ by the Jacobi polynomials converges pointwise and if $\frac{n^{4}}{N} \rightarrow 0$ as $n, N \rightarrow \infty$.

Proof

The proof follows directly from Theorems 1.1, 2.1 and 2.2 in Ref. [9].

\section{Operational matrices:}

Now, some explicit formulations for operational matrix of fractional integration, in the Riemann-Liouville sense, for the SDCPs will be obtained. Moreover, the product operational matrix for the SDCPs vector is defined. 
Lemma 1

For a positive number $r$ the inner product of the $\mathfrak{T}_{n, N}(t)$ and $t^{r}$, denoted by $\beta(n, r)$, can be derived as:

$$
\beta(n, r)=\frac{1}{N^{r}} \sum_{j=0}^{N} \sum_{k=0}^{n} a_{j, n} j^{k+r} .
$$

where $a_{j, n}$ is defined in relation (9).

Proof

By using definition of the discrete inner product $\langle., .\rangle_{*}$ in (11), we get:

$$
\beta(n, r)=\left\langle t^{r}, \mathfrak{T}_{n, N}(t)\right\rangle_{*}=\sum_{j=0}^{N} \mathfrak{T}_{n, N}\left(\frac{j}{N}\right) \frac{j^{r}}{N^{r}}=\frac{1}{N^{r}} \sum_{j=0}^{N} \mathcal{T}_{n, N}(j) j^{r} .
$$

Now, the analytical form of the discrete Chebyshev polynomial $\mathcal{T}_{n, N}$ in relation (8) results (16).

Theorem 2

Let $\Psi(t)$ be the SDCPs vector as defined in (14). Then its fractional integral of order $\nu$ in the Riemann-Liouville sense is given by

$$
{ }_{0} I_{t}^{\nu} \Psi(t)=\mathcal{P}^{(\nu)} \Psi(t)
$$

where $\mathcal{P}^{(\nu)}$ is the operational matrix of fractional integration and its $(i, j)$ th element can be derived as

$$
\mathcal{P}_{i, j}^{(\nu)}=\sum_{j=0}^{N}\left(\sum_{k=0}^{i} \frac{a_{k, N} \beta(k+\nu, j) \nu(k+1)}{\nu(k+\nu+1)}\right), \quad i, j=1,2, \ldots, N+1 .
$$

Proof

The $i$ th element of the vector $\Psi(t)$ is $\mathfrak{T}_{i-1, N}(t)$. Therefore, its fractional integration of order $\nu$ may be written as

$$
{ }_{0} I_{t}^{\nu} \mathfrak{T}_{i-1, N}(t)={ }_{0} I_{t}^{\nu} \sum_{k=0}^{i-1} N^{k} a_{k, i-1} t^{k}=\sum_{k=0}^{i-1} N^{k} a_{k, i-1}\left({ }_{0} I_{t}^{\nu} t^{k}\right)=\sum_{k=0}^{i-1} \frac{\Gamma(k+1) N^{k} a_{k, i-1}}{\Gamma(\nu+k+1)} t^{k+\nu} .
$$

Now by expanding the term $t^{k+\nu}$ we get

$$
t^{k+\nu} \simeq \sum_{j=0}^{N} b_{k, j} \mathfrak{T}_{j, N}(t)
$$

in which $b_{k, j}$ can be derived as

$$
b_{k, j}=\frac{\left\langle x^{k+\nu}, \mathfrak{T}_{j, N}(t)\right\rangle_{*}}{\left\langle\mathfrak{T}_{j, N}(t), \mathfrak{T}_{j, N}(t)\right\rangle_{*}}=\frac{\beta(k+\nu, j)}{\mu_{j}} .
$$

By putting Eqs. (21) and (20) in (19), we have:

$$
{ }_{0} I_{t}^{\nu} \mathfrak{T}_{i-1, N}(t)=\sum_{j=0}^{N}\left(\sum_{k=0}^{i-1} \frac{\beta(k+\nu, j) \Gamma(k+1) N^{k} a_{k, i-1}}{\mu_{j} \Gamma(\nu+k+1)}\right) \mathfrak{T}_{j, N}(t) .
$$

Accordingly, the desired result is derived. 


\section{Theorem 3}

Let $\Psi(t)$ be the SDCPs vector defined in (14) and $V$ be an arbitrary $(N+1)$ vector. Then, we can write

$$
\Psi(t) \Psi^{T}(t) V=\tilde{V} \Psi(t),
$$

where $\tilde{V}$ is the $(N+1) \times(N+1)$ product operational matrix and its $(i, j)$ th element can be defined as

$$
\tilde{V}_{i+1, j+1}=\frac{1}{\mu_{j}} \sum_{k=0}^{N} V_{k}\left\langle\mathfrak{T}_{k, N}(t) \mathfrak{T}_{i, N}(t), \mathfrak{T}_{j, N}(t)\right\rangle_{*}, \quad i, j=0,1, \ldots, N
$$

Proof

The product of two SDCPs vectors $\Psi(t)$ and $\Psi^{T}(t)$ is a $(N+1) \times(N+1)$ matrix as follow

$$
\Psi(t) \Psi^{T}(t)=\left[\begin{array}{cccc}
\mathfrak{T}_{0, N}(t) \mathfrak{T}_{0, N}(t) & \mathfrak{T}_{0, N}(t) \mathfrak{T}_{1, N}(t) & \ldots & \mathfrak{T}_{0, N}(t) \mathfrak{T}_{N, N}(t) \\
\mathfrak{T}_{1, N}(t) \mathfrak{T}_{0, N}(t) & \mathfrak{T}_{1, N}(t) \mathfrak{T}_{1, N}(t) & \ldots & \mathfrak{T}_{1, N}(t) \mathfrak{T}_{N, N}(t) \\
\vdots & \vdots & \ddots & \vdots \\
\mathfrak{T}_{N, N}(t) \mathfrak{T}_{0, N}(t) & \mathfrak{T}_{N, N}(t) \mathfrak{T}_{1, N}(t) & \ldots & \mathfrak{T}_{N, N}(t) \mathfrak{T}_{N, N}(t)
\end{array}\right]
$$

As a result, the relation (23) can be rewritten as:

$$
\sum_{k=0}^{N} \mathfrak{T}_{k, N}(t) \mathfrak{T}_{i, N}(t) V_{k+1}=\sum_{k=0}^{N} \mathfrak{T}_{j, N}(t) \tilde{V}_{i+1, k+1}, \quad i=0,1, \ldots, N .
$$

Now, by multiplying both sides of the above relation by $\mathfrak{T}_{j, N}(t)$ and using the defined inner product in (10), we get:

$$
\sum_{k=0}^{N}\left\langle\mathfrak{T}_{k, N}(t) \mathfrak{T}_{i, N}(t), \mathfrak{T}_{i, N}(t)\right\rangle_{*} V_{k+1}=\left\langle\mathfrak{T}_{j, N}(t), \mathfrak{T}_{j, N}(t)\right\rangle_{*} \tilde{V}_{i+1, j+1}, \quad i, j=0,1, \ldots, N
$$

Therefore, the matrix $\tilde{V}$ may be given by

$$
\tilde{V}_{i+1, j+1}=\frac{\sum_{k=0}^{N} V_{k}\left\langle\mathfrak{T}_{k, N}(t) \mathfrak{T}_{i, N}(t), \mathfrak{T}_{j, N}(t)\right\rangle_{*}}{\left\langle\mathfrak{T}_{j, N}(t), \mathfrak{T}_{j, N}(t)\right\rangle_{*}}=\frac{1}{\mu_{j}} \sum_{k=0}^{N} V_{k}\left\langle\mathfrak{T}_{k, N}(t) \mathfrak{T}_{i, N}(t), \mathfrak{T}_{j, N}(t)\right\rangle_{*} .
$$

\section{The numerical approach}

In this section, we will apply the SDCPs and thier operational matrix to solve the FVP (1)-(2) with boundary conditions (3). First, we approximate ${ }_{0}^{C} D_{t}^{\nu} x(t)$ via SDCPs as follows:

$$
{ }_{0}^{C} D_{t}^{\nu} x(t) \simeq C^{T} \Psi(t)
$$

where $\Psi(t)$ is the SDCPs vector as defined in relation (14) and $C$ is unknown coefficient vector defined as:

$$
C=\left(\begin{array}{l}
c_{1} \\
c_{2} \\
\vdots \\
c_{N}
\end{array}\right)
$$


By applying the fractional Riemann-Liouville operator ${ }_{0} I_{t}^{\nu}$ on both sides of the relation (24), we have:

$$
x(t) \simeq{ }_{0} I_{t}^{\nu} C^{T} \Psi(t)+\alpha=C^{T} \mathcal{P}^{(\nu)} \Psi(t)+d^{T} \Psi(t),
$$

in which $d$ is the SDCPs coefficient vector for $\alpha$ and $P^{(\nu)}$ is the operational matrix of fractional integration of the SDCPs vector $\Psi(t)$ derived in Theorem 2. So, the boundary condition in relation (3) can be written as follows:

$$
C^{T} \mathcal{P}^{(\nu)} \Psi(b)+d^{T} \Psi(b)-\beta \simeq 0,
$$

Moreover, by inserting the relations (24) and (26) the function $u(t)$ in (2) can be approximated as a function of unknown vector $C$, namely:

$$
u[t, C] \simeq \int_{a}^{t} \mathcal{K}\left(s, C^{T} \mathcal{P}^{\nu} \Psi(s)+d^{T} \Psi(s), C^{T} \Psi(s)\right) d s .
$$

Therefore, the performance index (1) can be derived as follows:

$$
J_{N}[C] \simeq \int_{a}^{b} \mathcal{F}\left(t, C^{T} \mathcal{P}^{\nu} \Psi(s)+d^{T} \Psi(s), C^{T} \Psi(s), u[t, C]\right) d t .
$$

Finally, by applying the necessary conditions for optimality of the performance index $J_{N}[C]$ we are able to write

$$
\frac{\partial J_{N}}{\partial c_{i}}=0, \quad i=0,1, \ldots, N-1 .
$$

Eqs. (27) along with (30) generate a set of $(N+1)$ nonlinear equations that can be solved by the Newton's iterative method to find the coefficients $c_{i}, i=0,1, \ldots, N$.

\section{Main features of the SDCPs method}

By using the SDCPs which are orthogonal with respect a discrete norm, a numerical scheme has been proposed to approximate solution of FVPs. The implementation of this method is more efficient and less complex in comparison with similare methods such as the classical Chebyshev polynomials method in which continuous norm is used. Moreover, the need for using Lagrange multiplier during the solution procedure is eliminated.

\section{Computational results and comparisons}

In order to verify the efficiency and superiority of the SDCPs method, we will consider some examples in this section. Let $e(t)=x(t)-\tilde{x}(t)$ be the error function of the approximate solution $\tilde{x}(t)$. The maximum absolute error (MAE) for the obtained numerical solution will be estimated as follow:

$$
\|e\|_{\infty}=\underset{0 \leq t \leq 1}{\operatorname{Max}}|e(t)| .
$$

All computations are carried out using MAPLE 17 with 30 digits precision.

\section{Example 1}

As first example, we consider the following FVP

$$
\operatorname{Min.} J(x)=\int_{0}^{1}\left[\left({ }_{0}^{C} D_{t}^{\nu} x(t)-t \Gamma(\nu+2)\right)^{2}+u(t)\right] d t, \quad 0<\nu \leq 1,
$$

in which

$$
u(t)=\int_{0}^{t}\left(x(s)-s^{\nu+1}\right)^{2} d s, \quad 0 \leq t \leq 1,
$$


subject to the boundary conditions

$$
x(0)=0, \quad x(1)=1 .
$$

The exact solution of this problem is $x(t)=t^{\nu+1}$. The proposed DCPs method has been applied to solve this FVP for various values $\nu$. Fig. 1 shows the obtained approximate solution $x(t)$ and the absolute error functions for different choices of $\nu$ and $N=10$. The MAE for the approximate solutions with different values of $N$ and $\nu$ are provided in Table 1. Moreover, in Table 2, the obtained optimal values $J$ and the required CPU time (in seconds) are compared with those achieved by the classical Chebyshev polynomials in Ref. [16]. From these results, we can conclude that both discrete and classical Chebyshev polynomials are efficient for solving this FVP, while the discrete polynomials method requires less CPU time when compared to the contentious one. Moreover, as the number of basis functions i.e. $N$ increases, complexity and required CPU time of the contentious Chebyshev polynomials method increases significantly.
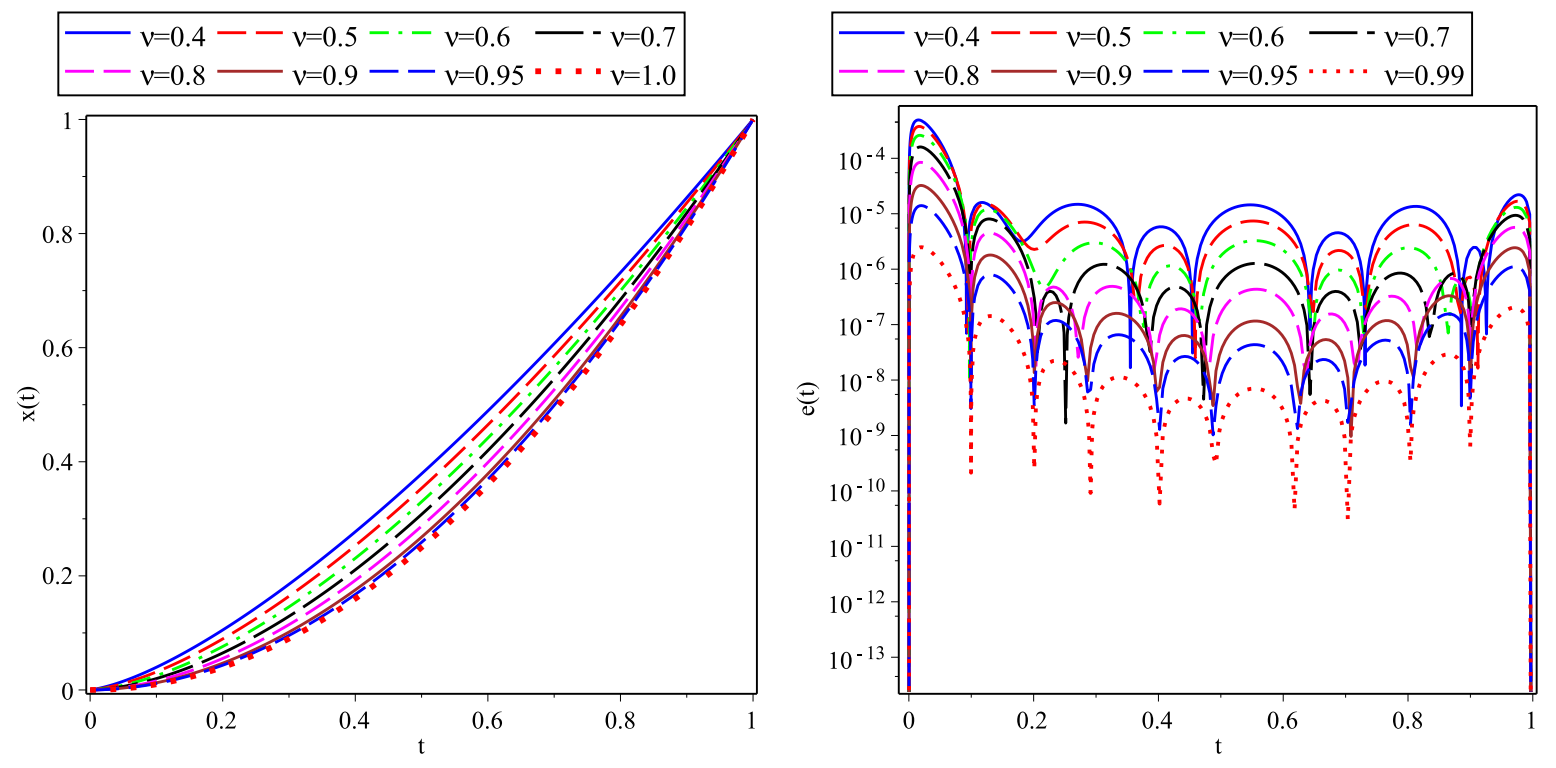

Figure 1. The obtained approximate solutions (Left) and the absolute error functions (Right) for different values of $\nu$ and $N=10$ (Example 1).

Table 1. The MAE of the approximate solution $\tilde{x}(t)$ (Example 1).

\begin{tabular}{cccc}
\hline$\nu$ & $N=6$ & $N=8$ & $N=12$ \\
\hline$\nu=0.50$ & $1.7 \times 10^{-3}$ & $6.2 \times 10^{-4}$ & $2.2 \times 10^{-4}$ \\
$\nu=0.70$ & $5.6 \times 10^{-4}$ & $2.5 \times 10^{-4}$ & $1.0 \times 10^{-4}$ \\
$\nu=0.80$ & $3.3 \times 10^{-4}$ & $1.4 \times 10^{-4}$ & $5.1 \times 10^{-5}$ \\
$\nu=0.90$ & $1.2 \times 10^{-4}$ & $5.3 \times 10^{-5}$ & $1.8 \times 10^{-5}$ \\
$\nu=0.95$ & $5.8 \times 10^{-5}$ & $2.5 \times 10^{-5}$ & $8.2 \times 10^{-6}$ \\
$\nu=0.99$ & $1.2 \times 10^{-5}$ & $4.5 \times 10^{-6}$ & $1.4 \times 10^{-6}$ \\
\hline
\end{tabular}

Example 2

Now, we consider the following FVP

$$
\operatorname{Min.} J(x)=\int_{0}^{1}\left[\left({ }_{0}^{C} D_{t}^{\nu} x(t)-1\right)^{2}+u(t)\right] d t, 0<\nu \leq 1,
$$


Table 2. The optimal values $J$ and required CPU time (in seconds) for different values of $\nu$ and $N$ (Example 1).

\begin{tabular}{|c|c|c|c|c|}
\hline & \multicolumn{2}{|c|}{$N=6$} & & \\
\hline & \multicolumn{2}{|l|}{$J$} & \multicolumn{2}{|l|}{ CPU time } \\
\hline & Chebyshev polynomial [16] & DCPs & Chebyshev polynomials & DCPs \\
\hline$\nu=0.50$ & $7.677 \times 10^{-9}$ & $1.052 \times 10^{-8}$ & 8.078 & 2.469 \\
\hline$\nu=0.70$ & $3.329 \times 10^{-9}$ & $2.175 \times 10^{-8}$ & 8.063 & 2.343 \\
\hline$\nu=0.90$ & $2.588 \times 10^{-10}$ & $9.133 \times 10^{-10}$ & 8.063 & 2.782 \\
\hline \multirow[t]{4}{*}{$\nu=0.99$} & $1.986 \times 10^{-12}$ & $7.811 \times 10^{-12}$ & 8.297 & 2.438 \\
\hline & \multicolumn{2}{|c|}{$N=8$} & & \\
\hline & \multicolumn{2}{|l|}{$J$} & \multicolumn{2}{|l|}{ CPU time } \\
\hline & Chebyshev polynomial [16] & DCPs & Chebyshev polynomials & DCPs \\
\hline$\nu=0.50$ & $8.338 \times 10^{-10}$ & $2.246 \times 10^{-9}$ & 17.203 & 4.735 \\
\hline$\nu=0.70$ & $3.076 \times 10^{-10}$ & $3.785 \times 10^{-10}$ & 18.843 & 4.641 \\
\hline$\nu=0.90$ & $1.928 \times 10^{-11}$ & $1.631 \times 10^{-11}$ & 17.688 & 4.454 \\
\hline \multirow[t]{4}{*}{$\nu=0.99$} & $1.336 \times 10^{-13}$ & $1.034 \times 10^{-13}$ & 18.828 & 4.703 \\
\hline & \multicolumn{2}{|c|}{$N=10$} & & \\
\hline & \multicolumn{2}{|l|}{$J$} & \multicolumn{2}{|l|}{ CPU time } \\
\hline & Chebyshev polynomial [16] & DCPs & Chebyshev polynomials & DCPs \\
\hline$\nu=0.50$ & $1.458 \times 10^{-10}$ & $7.286 \times 10^{-9}$ & 25.891 & 7.500 \\
\hline$\nu=0.70$ & $4.739 \times 10^{-11}$ & $1.021 \times 10^{-10}$ & 26.765 & 9.609 \\
\hline$\nu=0.90$ & $2.512 \times 10^{-12}$ & $3.861 \times 10^{-12}$ & 25.500 & 9.547 \\
\hline$\nu=0.99$ & $1.608 \times 10^{-14}$ & $2.258 \times 10^{-13}$ & 26.063 & 9.625 \\
\hline
\end{tabular}

in which

$$
u(t)=\int_{0}^{t}\left(x(s)-\frac{s^{\nu+1}}{\Gamma(\nu+2)}\right)^{2} d s, \quad 0 \leq t \leq 1,
$$

subject to the boundary conditions

$$
x(0)=1, x(1)=\frac{1}{\Gamma(\nu+1)} .
$$

The exact solution is $x(t)=\frac{t^{\nu}}{\Gamma(\nu+1)}$. The described DCPs method in Section 5 with different values of $N$ and $\nu$ have been applied to solve this problem. The approximate solutions $x(t)$ and their absolute error functions for $N=10$ and variouse values of $\nu$ are plotted in Fig. 2. Moreover, the MAE of the approximate solution $\tilde{x}(t)$ for variouse values of $N$ and $\nu$ are listed in Table 3. In order to verify the superiority and efficiency of the DCPs method, in Table 4 the obtained optimal values $J$ and the required CPU time (in seconds) were compared to those achieved by the classical Chebyshev polynomials in Ref. [16]. From these results, it is clear that the proposed DCPs method is efficient and accurate for solving FVPs. In addition, it can be concluded that application of DCPs is less time consuming and complex in comparison with the classical Chebyshev polynomials.

\section{Example 3}

Consider the following FVPs

$$
\operatorname{Min.} J(x)=\int_{0}^{1}\left[2\left({ }_{0}^{C} D_{t}^{\nu} x(t)-\frac{\Gamma(\nu+4)}{\Gamma(4)} t^{3}-\frac{\Gamma(\nu+3)}{\Gamma(3)} t^{2}\right)^{2}+5 u(t)\right] d t, \quad 0<\nu \leq 1,
$$



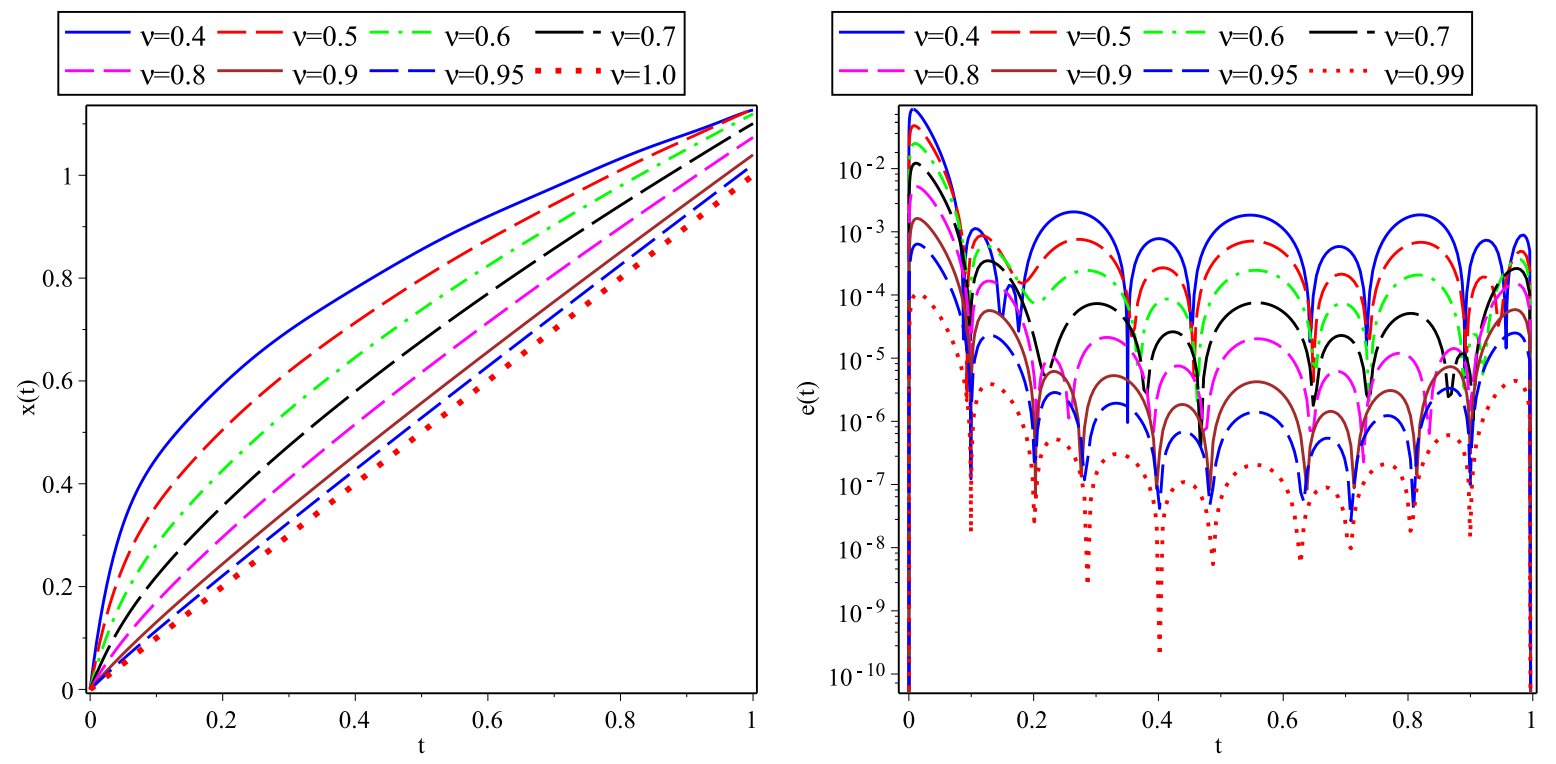

Figure 2. The obtained approximate solutions (Left) and the absolute error functions (Right) for different values of $\nu$ and $N=10$ (Example 2).

Table 3. The MAE of the approximate solution $\tilde{x}(t)$ (Example 2).

\begin{tabular}{cccc}
\hline$\nu$ & $N=8$ & $N=10$ & $N=12$ \\
\hline$\nu=0.50$ & $5.7 \times 10^{-2}$ & $8.7 \times 10^{-2}$ & $4.1 \times 10^{-2}$ \\
$\nu=0.70$ & $1.5 \times 10^{-2}$ & $1.2 \times 10^{-2}$ & $1.0 \times 10^{-2}$ \\
$\nu=0.80$ & $6.6 \times 10^{-3}$ & $5.1 \times 10^{-3}$ & $4.2 \times 10^{-3}$ \\
$\nu=0.90$ & $2.3 \times 10^{-3}$ & $1.6 \times 10^{-3}$ & $1.2 \times 10^{-3}$ \\
$\nu=0.95$ & $8.6 \times 10^{-4}$ & $6.3 \times 10^{-4}$ & $5.1 \times 10^{-4}$ \\
$\nu=0.99$ & $1.4 \times 10^{-4}$ & $1.1 \times 10^{-4}$ & $8.3 \times 10^{-5}$ \\
\hline
\end{tabular}

in which

$$
u(t)=\int_{0}^{t}\left(x(s)-s^{\nu+3}-s^{\nu+2}\right)^{2} d s, \quad 0 \leq t \leq 1,
$$

subject to the boundary conditions

$$
x(0)=0, x(1)=2, \quad 0 \leq t \leq 1 .
$$

For $0<\nu \leq 1$ the exact solution of this problem is $x(t)=t^{\nu+3}+t^{\nu+2}$. The solution of this FVP has been approximated by using the proposed DCPs method for variouse values of $N$ and $\nu$. The approximate solutions $\tilde{x}(t)$ and their absolute error functions for $N=10$ and variouse values of $\nu$ are plotted in Fig. 3. The MAE of the solution functions $x(t)$ for different choices of $N$ and $\nu$ are presented in Table 5. Furthermore, Table 6 provides a comparison between the obtained optimal values $J$ and their required CPU time (in seconds) and those derived by the classical Chebyshev polynomials in Ref. [16]. According to the presented results, it is clear that both DCPs and classical Chebyshev polynomials methods are efficient in solving FVPs, while application of DCPs is less time consuming and complex in comparison to the typical Chebyshev polynomials method. Moreover, it is easy to conclude that complexity and required CPU time of the classical Chebyshev polynomials method increses significantly as the number of basis functions $N$ increases. 
Table 4. The optimal values $J$ and required CPU time (in seconds) for different values of $\nu$ and $N$ (Example 2).

\begin{tabular}{|c|c|c|c|c|}
\hline & \multicolumn{2}{|c|}{$N=8$} & \multirow{2}{*}{\multicolumn{2}{|c|}{ CPU time }} \\
\hline & \multicolumn{2}{|l|}{$J$} & & \\
\hline & Chebyshev polynomial [16] & DCPs & Chebyshev polynomials & DCPs \\
\hline$\nu=0.50$ & $2.427 \times 10^{-6}$ & $1.19 \times 10^{-5}$ & 28.578 & 9.344 \\
\hline$\nu=0.70$ & $4.548 \times 10^{-7}$ & $9.195 \times 10^{-7}$ & 29.250 & 9.922 \\
\hline \multirow{4}{*}{$\nu=0.99$} & $7.611 \times 10^{-11}$ & $8.553 \times 10^{-11}$ & 29.094 & 10.454 \\
\hline & \multicolumn{2}{|c|}{$N=10$} & & \\
\hline & \multicolumn{2}{|l|}{$J$} & \multicolumn{2}{|l|}{ CPU time } \\
\hline & Chebyshev polynomial [16] & DCPs & Chebyshev polynomials & DCPs \\
\hline$\nu=0.50$ & $9.933 \times 10^{-7}$ & $6.476 \times 10^{-6}$ & 59.110 & 19.531 \\
\hline$\nu=0.70$ & $1.647 \times 10^{-7}$ & $4.533 \times 10^{-7}$ & 58.453 & 20.922 \\
\hline \multirow[t]{4}{*}{$\nu=0.99$} & $2.171 \times 10^{-11}$ & $3.573 \times 10^{-11}$ & 57.829 & 21.093 \\
\hline & \multicolumn{2}{|c|}{$N=12$} & & \\
\hline & \multicolumn{2}{|l|}{$J$} & \multicolumn{2}{|l|}{ CPU time } \\
\hline & Chebyshev polynomial [16] & DCPs & Chebyshev polynomials & DCPs \\
\hline & $4.845 \times 10^{-7}$ & $3.751 \times 10^{-6}$ & 82.532 & 32.640 \\
\hline$\nu=0.70$ & $7.166 \times 10^{-7}$ & $9.504 \times 10^{-7}$ & 96.938 & 38.031 \\
\hline$\nu=0.99$ & $7.713 \times 10^{-12}$ & $1.760 \times 10^{-12}$ & 98.297 & 39.921 \\
\hline
\end{tabular}
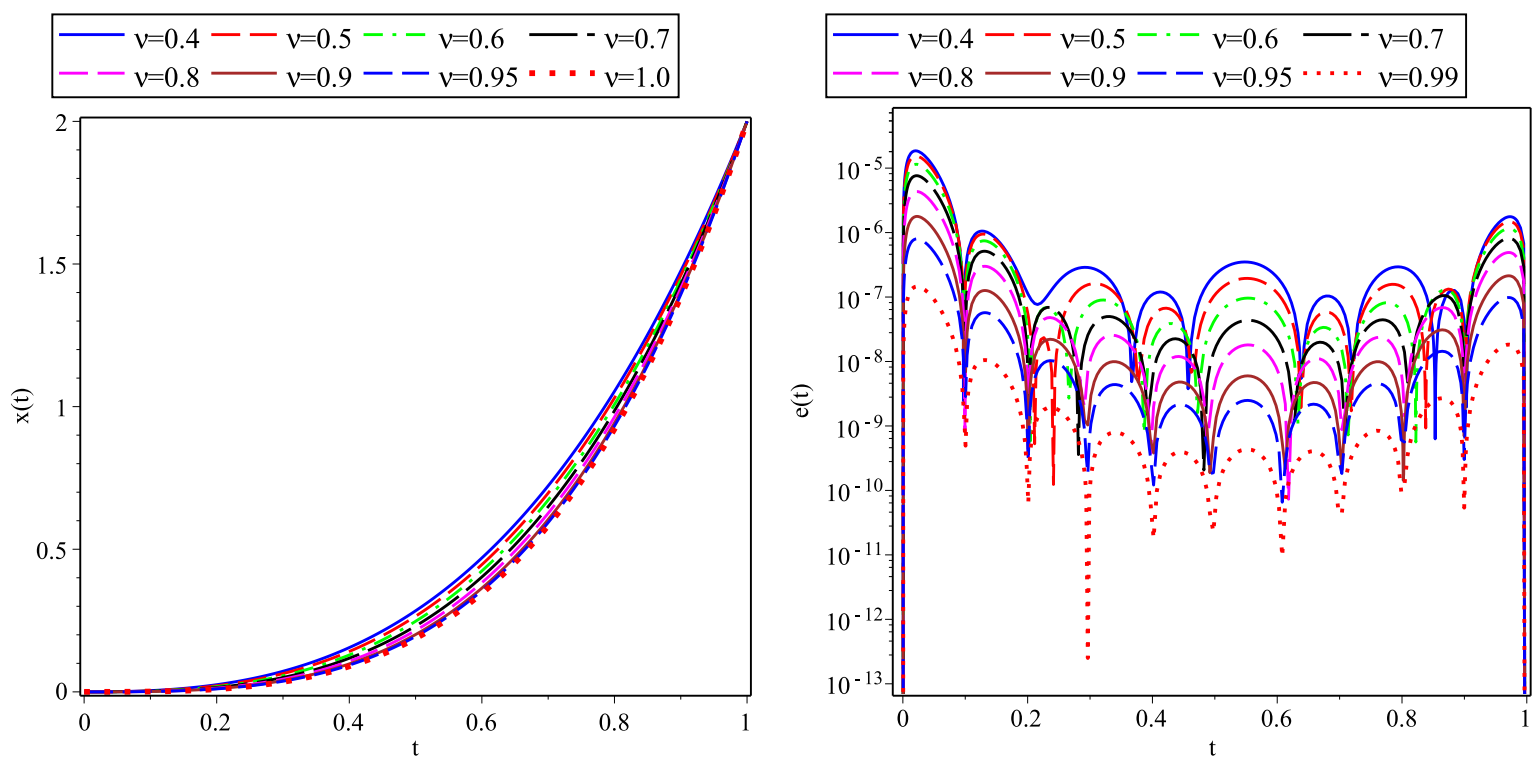

Figure 3. The obtained approximate solutions (Left) and the absolute error functions (Right) for different values of $\nu$ and $N=10$ (Example 3). 
Table 5. The MAE of the approximate solution $\tilde{x}(t)$ (Example 3).

\begin{tabular}{cccc}
\hline & $N=6$ & $N=8$ & $N=12$ \\
\hline$\nu=0.50$ & $8.7 \times 10^{-5}$ & $3.7 \times 10^{-5}$ & $7.7 \times 10^{-6}$ \\
$\nu=0.70$ & $4.8 \times 10^{-5}$ & $1.6 \times 10^{-5}$ & $3.9 \times 10^{-6}$ \\
$\nu=0.80$ & $2.9 \times 10^{-5}$ & $1.1 \times 10^{-5}$ & $2.3 \times 10^{-6}$ \\
$\nu=0.90$ & $1.2 \times 10^{-5}$ & $4.0 \times 10^{-6}$ & $2.2 \times 10^{-7}$ \\
$\nu=0.95$ & $5.6 \times 10^{-6}$ & $1.8 \times 10^{-6}$ & $3.8 \times 10^{-7}$ \\
$\nu=0.99$ & $1.6 \times 10^{-7}$ & $3.8 \times 10^{-7}$ & $6.8 \times 10^{-8}$ \\
\hline
\end{tabular}

Table 6. The optimal values $J$ and required CPU time (in seconds) for different values of $\nu$ and $N$ (Example 3).

\begin{tabular}{|c|c|c|c|c|}
\hline & \multicolumn{2}{|c|}{$N=5$} & & \\
\hline & \multicolumn{2}{|l|}{$J$} & \multicolumn{2}{|l|}{ CPU time } \\
\hline & Chebyshev polynomials [16] & DCPs & Chebyshev polynomials & DCPs \\
\hline$\nu=0.5$ & $2.855 \times 10^{-10}$ & $1.960 \times 10^{-9}$ & 17.109 & 3.438 \\
\hline$\nu=0.7$ & $1.366 \times 10^{-10}$ & $5.882 \times 10^{-10}$ & 17.875 & 3.438 \\
\hline \multirow[t]{4}{*}{$\nu=0.9$} & $9.337 \times 10^{-12}$ & $3.740 \times 10^{-11}$ & 18.922 & 3.406 \\
\hline & \multicolumn{2}{|c|}{$N=7$} & & \\
\hline & \multicolumn{2}{|l|}{$J$} & \multicolumn{2}{|l|}{ CPU time } \\
\hline & Chebyshev polynomials [16] & DCPs & Chebyshev polynomials & DCPs \\
\hline$\nu=0.5$ & $1.291 \times 10^{-11}$ & $1.615 \times 10^{-10}$ & 24.344 & 5.922 \\
\hline$\nu=0.7$ & $6.855 \times 10^{-12}$ & $4.592 \times 10^{-11}$ & 24.344 & 6.484 \\
\hline \multirow[t]{4}{*}{$\nu=0.9$} & $6.061 \times 10^{-13}$ & $3.001 \times 10^{-12}$ & 24.125 & 6.078 \\
\hline & \multicolumn{2}{|c|}{$N=9$} & & \\
\hline & \multicolumn{2}{|l|}{$J$} & \multicolumn{2}{|l|}{ CPU time } \\
\hline & Chebyshev polynomials [16] & DCPs & Chebyshev polynomials & DCPs \\
\hline$\nu=0.5$ & $8.479 \times 10^{-13}$ & $2.156 \times 10^{-12}$ & 68.532 & 10.750 \\
\hline$\nu=0.7$ & $4.035 \times 10^{-13}$ & $5.438 \times 10^{-12}$ & 64.797 & 11.312 \\
\hline$\nu=0.9$ & $3.089 \times 10^{-14}$ & $3.127 \times 10^{-13}$ & 64.891 & 11.235 \\
\hline
\end{tabular}

\section{Concluding Remarks}

This paper proposed a new type of discrete orthogonal polynomials basis. By using these orthogonal polynomials and their fractional operational matrix a numerical method is developed to solve FVPs. As these polynomials are orthogonal with respect to a discrete norm, implementation of the presented method is more efficient and less complex in comparison with similar methods in which continuous orthogonal polynomials are used. Application of the proposed method for some test problems verified that:

1. The discrete orthogonal polynomials are efficient for solving FVPs.

2. Application of discrete Chebyshev polynomials is less time consuming and complex in comparison with the classical Chebyshev polynomials.

3. The complexity and required CPU time of classical Chebyshev polynomials increases significantly as the number of basis functions increases.

4. The need for using Lagrange multiplier during the solution procedure is eliminated. 


\section{REFERENCES}

1. P. K. Sahu, and S. S. Ray, Comparison for accurate solutions of nonlinear Hammerstein fuzzy integral equations, Mathematical Communications, vol. 21, no. 2, pp. 283-299, 2016.

2. F. Mohammadi, S. T. Mohyud-Din, A fractional-order Legendre collocation method for solving the Bagley-Torvik equations. Adv. Differ. Equ. vol. 269, pp. 269, 2016.

3. H. Khosravian-Arab, R. Almeida, Numerical solution for fractional variational problems using the Jacobi polynomials, Appl Math Model. vol. 39, no. 21, pp. 6461-6470, 2015.

4. S. S. Ezz-Eldien, R. M. Hafez, A. H. Bhrawy, D. Baleanu, A. A. El-Kalaawy, New numerical approach for fractional variational problems using shifted Legendre orthonormal polynomials, J. Optimiz. Theory App., vol. 174, no. 1, pp. 295-320, 2017.

5. F. Mohammadi, C. Cattani, A generalized fractional-order Legendre wavelet Tau method for solving fractional differential equations, J. Comput. Appl. Math., 2017.

6. M. A. Zaky, E. H. Doha, T. M. Taha, and D. Baleanu, New recursive approximations for variable-order fractional operators with applications, Mathematical Modelling and Analysis, vol. 23, no. 2, pp. 227-239, 2018.

7. M. A. Zaky, A Legendre spectral quadrature tau method for the multi-term time-fractional diffusion equations, Comp. Appl. Math. vol. 37, pp. 3525-3538, 2018.

8. D. Xiu, G. E. Karniadakis, The Wiener-Askey polynomial chaos for stochastic differential equations, SIAM journal on scientific computing, vol. 24, no. 2, pp. 619-644, 2002.

9. R. Goertz, P. Öffner, On Hahn polynomial expansion of a continuous function of bounded variation, ArXiv e-prints: arXiv:1610.06748, 2016.

10. B. H. S. Asli, J. Flusser, New discrete orthogonal moments for signal analysis, Signal Processing, vol. 141, pp. 57-73, 2017.

11. R. Almeida, D. F.M. Torres, Calculus of variations with fractional derivatives and fractional integrals, Appl. Math. Lett. vol. 22, no. 12, pp.1816-1820, 2009.

12. A. Malinowska, D. Torres, Fractional calculus of variations for a combined Caputo derivative, Fract. Calc. Appl. Anal. vol. 14, no. 4, pp. 523-537, 2011.

13. B. van Brunt, The Calculus of Variations, Springer-Verlag, New York, 2004.

14. M. A. Zaky, and J. T. Machado, On the formulation and numerical simulation of distributed-order fractional optimal control problems, Commun. Nonlinear. Sci. Numer. Simul., vol. 52, pp. 177-189, 2017.

15. D. Baleanu, S.I. Muslih, Lagrangian formulation of classical fields within Riemann-Liouville fractional derivatives, Phys. Scripta, vol. 72, no. 3, pp.119-121, 2005.

16. S.S. Ezz-Eldien, E.H. Doha, A.H. Bhrawy, A.A. El-Kalaawy, J.A.T. Machado, A new operational approach for solving fractional variational problems depending on indefinite integrals, Commun Nonlinear Sci Numer Simul. vol. 57, pp. 246-263, 2018.

17. A. B. Malinowska, T. Odzijewicz, D. F. Torres, Advanced methods in the fractional calculus of variations, Springer, 2015.

18. F. Riewe, Nonconservative Lagrangian and Hamiltonian mechanics, Phys. Rev. E., vol. 53, no. 2, pp. 1890-1899, 1996.

19. F. Riewe, Mechanics with fractional derivatives, Phys. Rev. E., vol. 55, no. 3, pp. 3581-3592, 1997.

20. I. Malmir, Optimal control of linear time-varying systems with state and input delays by Chebyshev wavelets, Statistics, Optimization \& Information Computing, vol. 5, no. 4, pp. 302-324 2017. http://dx.doi.org/10.19139/soic.v5i4.341

21. M. Barrios, G. Reyero, An Euler-Lagrange Equation only Depending on Derivatives of Caputo for Fractional Variational Problems with Classical Derivatives, Statistics, Optimization \& Information Computing, vol. 8, no. 2, pp. 590C601, 2020. https://doi.org/10.19139/soic-2310-5070-865

22. S. G. Samko, A.A. Kilbas, O. I. Marichev, Fractional Integrals and Derivatives: Theory and Applications, Gordon and Breach, Langhorne, 1993.

23. S. S. Ray, A. Atangana, S. C. Noutchie, M. Kurulay, N. Bildik and A. Kilicman, Fractional calculus and its applications in applied mathematics and other sciences, Mathematical Problems in Engineering, 2014.

24. I. Podlubny, Fractional Differential Equations, Academic Press, San Diego, 1999.

25. N. Gogin and M. Hirvensalo, On the generating function of Discrete Chebyshev Polynomials, Journal of Mathematical Sciences, vol. 224, no. 2, 2017.

26. A. F. Nikiforov, S. K. Suslov and V. B. Uvarov, Classical orthogonal polynomials of a discrete variable, Springer Berlin Heidelberg, 1991.

27. H.M. Srivastava and J. Choi, Series Associated with the Zeta and Related Functions, Kluwer, Boston, 2001.

28. J. Riordan, An Introduction to Combinatorial Analysis, New York: Wiley, 1980. 